Reprod. Nutr. Dévelop., 1980, 20 (5 B), 1685-1689.

\title{
Effet du mode de distribution du foin et des céréales sur l'activité cellulolytique dans le gros intestin du poney
}

\author{
par J. L. TISSERAND, M. OTTIN PECCHIO, G. ROLLIN \\ avec la collaboration technique de Catherine CORDELET \\ Laboratoire de Recherches de la Chaire de Zootechnie \\ ENSSAA-INRA, 26, Bd Dr Petitjean, 21100 Dijon, France.
}

Summary. Effect of distributing hay and cereals on cellulolytic activity in the large intestine of the pony.

The cellulolytic activity in the large intestine of the pony varies according to the form and the composition of the feed.

This activity was measured on two caecal and ventral colon-cannulated ponies receiving the following 4 diets during four successive 6-week periods :

$-6 \mathrm{~kg}$ of hay,

$-4 \mathrm{~kg}$ of hay $+1 \mathrm{~kg}$ of oats,

- $6 \mathrm{~kg}$ of ground, pelleted hay,

- $5 \mathrm{~kg}$ of a ground, pelleted blend of 80 p. 100 hay and 20 p. 100 oats.

Adding oats to a hay feed increased the cellulolytical activity in the caecum and the colon, whereas grinding and pelleting hay alone or hay enriched with oats diminished that activity in the caecum without modifying it in the colon (table 1).

The volatile fatty acid production shown in table 2 does not always vary in the same way as cellulolytic activity; this might be due to some variations in the values of the digestive contents in the two compartments.

\section{Introduction.}

L'utilisation des fourrages est fonction de l'activité microbienne caractérisant la digestion dans le gros intestin des équidés (Alexander, 1972 ; Argenzio, Southworth et Stevens, 1974 ; Hintz et al., 1971 ; Robinson et Slade, 1974 ; Tisserand, 1979). Celle-ci est particulièrement importante dans le cæcum et elle se poursuit avec une efficacité moindre dans la fraction ventrale du côlon (Kern et al., 1973 ; Kolb ef Gurtler, 1971 ; Tisserand et al., 1977a).

La dégradation de la cellulose dans le gros intestin varie en fonction de :

- l'intensité de la digestion des aliments dans l'intestin grêle qui est influencée par le transit rapide de la fraction des aliments qui passe directement dans le duodénum du fait du faible volume de l'estomac de cette espèce (Tisserand et Masson, 1976) ; - de la durée de séjour des aliments dans le cæcum qui est facteur de la taille des particules (Wolter, 1975). 
C'est pourquoi nous nous sommes demandés si le mode de présentation (sous forme longue ou agglomérée après broyage) d'une ration composée de foin ou de foin et de céréales pouvait influer sur l'intensité de la digestion de la cellulose dans le gros intestin et sur sa répartition entre le cæcum et le côlon ventral.

\section{Matériel et méthodes.}

Les animaux. - L'étude est effectuée sur deux poneys mâles Alezan (A) âgé de 5 ans, et Fanfan (F) âgé de 7 ans pesant respectivement 175 et $160 \mathrm{~kg}$ porteurs de deux canules chacun, l'une du cæcum, l'autre du côlon ventral droit (F) ou gauche (A) mises en place par M. Candau et C. Masson (Tisserand ef al., 1977b).

Les régimes alimentaires. - Quatre régimes sont expérimentés sur les deux sujets au cours de quatre périodes successives de 6 semaines chacune :

1. $-6 \mathrm{~kg}$ de foin de pré,

2. $-4 \mathrm{~kg}$ de foin de pré et $1 \mathrm{~kg}$ d'avoine,

3. $-6 \mathrm{~kg}$ de foin de pré broyé et aggloméré,

4. - $5 \mathrm{~kg}$ de mélange de foin de pré $(80$ p. 100$)$ et d'avoine (20 p. 100) broyé et aggloméré.

La ration est distribuée en deux repas par jour avec de l'eau ad libitum.

Mesures effectuées. - Après deux semaines d'accoutumance l'activité cellulolytique est évaluée durant quatre semaines, par la technique des sacs de nylon avec une mesure hebdomadaire d'une durée de $24 \mathrm{~h}$ dans le cæcum ef de $48 \mathrm{~h}$ dans le côlon. La dégradation de la cellulose du foin de pré expérimental y est déterminée par le dosage selon la méthode Van Soest. Chaque semaine de la période expérimentale trois prélèvements sont effectués le même jour à $10 \mathrm{~h}, 14 \mathrm{~h}$, et $17 \mathrm{~h} 30$ dans le cæcum et le côlon de chaque poney.

A chaque prélèvement $100 \mathrm{~cm}^{3}$ environ du contenu du cæcum ou du côlon sont obtenus par aspiration à l'aide d'une sonde en matière plastique introduite par la canule ef enveloppée dans un sac de toile nylon pour éviter de blesser l'animal. Sur ces échantillons les dosages suivants sont effectués :

- le $\mathrm{pH}$;

- l'azote ammoniacal par la méthode du Conway;

- I'azote total par la méthode de Kjeldahl;

- les acides gras volatils totaux et les proportions d'acides acétique, propionique et butyrique séparés par chromatographie en phase gazeuse (support chromosorb W 80-100 m phase stationnaire NPGA 25 p. 100 + acide phosphorique 2 p. 100).

Les moyennes par jour et par compartiment digestif sont établies pour chaque sujet.

\section{Résultats.}

Les valeurs moyennes des quatre mesures par régime et par poney sont rassemblées dans le tableau 1 . Sa lecture ne permet pas de comparer l'activité cellulolyti- 
que dans le cæcum et dans le côlon ventral puisque le temps de séjour est doublé dans ce dernier compartiment.

\section{TABLEAU 1}

Effet du régime sur l'activité cellulolytique dans le gros intestin du poney (résultał par poney)

\begin{tabular}{|c|c|c|c|c|}
\hline & Foin de pré & $\begin{array}{l}\text { Foin de pré } \\
+ \text { avoine }\end{array}$ & $\begin{array}{l}\text { Foin de pré } \\
\text { broyé } \\
\text { et aggloméré }\end{array}$ & $\begin{array}{l}\text { Foin de pré }+ \\
\text { avoine broyés } \\
\text { et agglomérés }\end{array}$ \\
\hline \multicolumn{5}{|c|}{ I. - Cœcum (Durée de l'essai : 24 h) } \\
\hline $\begin{array}{l}\text { Alezan } \ldots . . \\
\text { Fanfan } \\
\ldots\end{array}$ & $\begin{array}{l}23,9 \\
20,4\end{array}$ & $\begin{array}{l}25,9 \\
32,7\end{array}$ & $\begin{array}{l}18,2 \\
20,7\end{array}$ & $\begin{array}{l}14,6 \\
14,0\end{array}$ \\
\hline Moyenne .... & 22,1 & 29,3 & 19,5 & 14,3 \\
\hline \multicolumn{5}{|c|}{ II. - Côlon (Durée de l'essai : 48 h) } \\
\hline $\begin{array}{l}\text { Alezan } \ldots \ldots \\
\text { Fanfan } \ldots . .\end{array}$ & $\begin{array}{l}31,6 \\
34,0\end{array}$ & $\begin{array}{l}36,6 \\
42,3\end{array}$ & $\begin{array}{l}33,7 \\
28,6\end{array}$ & $\begin{array}{l}34,2 \\
35,6\end{array}$ \\
\hline Moyenne .... & 32,8 & 39,5 & 31,1 & 34,9 \\
\hline
\end{tabular}

Dans le cæcum, l'addition d'aroine à une ration de foin augmente sensiblement l'activité cellulolytique $(+30$ p. 100). Par contre le broyage et l'agglomération du foin n'entraînent qu'une faible diminution de l'activité cellulolytique observée sur un sujet (A) seulement; alors que le broyage ef l'agglomération du foin el de l'avoine ensemble provoquent une diminution de la cellulolyse (-35 p. 100). Dans le côlon l'addition d'avoine à la ration de foin augmente aussi l'activité cellulolytique

\section{TABLEAU 2}

Effet du régime sur certaines données biochimiques du cæcum et du côlon du poney (moyenne des 2 poneys)

\begin{tabular}{|c|c|c|c|c|}
\hline & Foin de pré & $\begin{array}{l}\text { Foin de } \\
\text { pré } \\
+ \text { avoine }\end{array}$ & $\begin{array}{l}\text { Foin de pré } \\
\text { broyé } \\
\text { et aggloméré }\end{array}$ & $\begin{array}{l}\text { Foin de pré } \\
\text { broyé ef } \\
\text { aggloméré }\end{array}$ \\
\hline $\begin{array}{l}\text { I. }- \text { Cæcum } \\
\text { pH } \ldots \ldots \ldots \ldots \ldots \ldots \\
\mathrm{NH}_{3} \mathrm{~N}_{\mathrm{T}}(\mathrm{p} .100) \ldots \ldots \ldots \\
\text { AGV totaux (mmoles/l) ... } \\
\text { Acide acétique }(\mathrm{p} .100) \ldots \\
\text { Acide propionique }(p .100) \\
\text { Acide butyrique }(\mathrm{p} .100) \ldots\end{array}$ & $\begin{array}{l}7,29 \\
6,7 \\
63 \\
78,7 \\
16,5 \\
4,2\end{array}$ & $\begin{array}{l}7,42 \\
5,9 \\
68 \\
78,5 \\
17,3 \\
3,7\end{array}$ & $\begin{array}{l}7,29 \\
4,4 \\
70 \\
78,6 \\
17,3 \\
3,8\end{array}$ & $\begin{array}{l}7,30 \\
5,9 \\
73 \\
79,4 \\
16,0 \\
4,1\end{array}$ \\
\hline $\begin{array}{l}\text { II. }- \text { Côlon } \\
\text { pH } \ldots \ldots \ldots \ldots \\
\mathrm{NH}_{3} / \mathrm{N}_{\mathrm{T}}(\mathrm{p} .100) \ldots \ldots \ldots \\
\text { AGV totaux (mmoles } / 1) \ldots \\
\text { Acide acétique (p. 100) } \ldots \\
\text { Acide propionique }(\mathrm{p} .100) \\
\text { Acide butyrique (p. 100) .. }\end{array}$ & $\begin{array}{l}7,45 \\
13,2 \\
70 \\
76,5 \\
16,9 \\
4,3\end{array}$ & $\begin{array}{l}7,53 \\
12,8 \\
70 \\
77,5 \\
16,2 \\
4,2\end{array}$ & $\begin{array}{l}7,50 \\
9,6 \\
73 \\
78,1 \\
16,0 \\
4,3\end{array}$ & $\begin{array}{l}7,50 \\
11,2 \\
77 \\
78,7 \\
14,8 \\
4,4\end{array}$ \\
\hline
\end{tabular}


$(+20$ p. 100) mais l'agglomération du foin seul ou avec l'avoine ne modifie pratiquement pas l'activité cellulolytique.

La lecture du tableau 2 qui renferme les données biochimiques observées pour chaque régime dans le cæcum et le côlon montre que :

- le régime n'entraîne aucune différence notable de $\mathrm{pH}$ dans le cæcum et dans le côlon ventral ;

- l'addition de céréales ou le broyage de la ration provoquent dans le cæcum une diminution du pourcentage d'azote ammoniacal et une augmentation de la concentration en acides gras volatils totaux sans toutefois modifier sensiblement les proportions molaires des acides acétique, propionique ef butyrique;

- dans le côlon ventral, l'agglomération de la ration entraîne une légère augmentation de la proportion molaire d'acide acétique au détriment de celle d'acide propionique.

\section{Discussion et conclusion.}

Lorsque l'avoine ef le foin sont mis à la disposition des animaux en même temps, ceux-ci ont tendance à consommer l'avoine en premier, qui, chassée par le fourrage ingéré ultérieurement, transite rapidement dans l'intestin grêle ef y est incomplètement digéré. II s'ensuit une stimulation de l'activité microbienne consécutive à l'arrivée d'amidon dans le cæcum qui pourrait expliquer l'augmentation de l'activité cellulolytique.

Par contre la distribution de foin et d'avoine agglomérés ensemble entraîne une diminution du volume permettant le séjour des aliments dans l'estomac, un transit lent et une digestion plus complète dans l'intestin grêle ce qui pourrait expliquer une baisse de l'activité cellulolytique constatée dans le cæcum. De plus la présence de particules fines dans l'aliment limite le séjour des aliments cellulosiques dans le cæcum et favorise leur passage direct dans le côlon, d'où le maintien de l'activité cellulolytique dans ce compartiment. II convient d'être très prudent dans l'interprétation des données biochimiques correspondant à des concentrations variant avec de nombreux facteurs et en particulier le volume des contenus de côlon et de cæcum. En particulier, une diminution du volume du contenu du cæcum avec les rations agglomérées pourrait y expliquer l'augmentation des concentrations en acides gras volatils alors que la dégradation de la cellulose diminue.

II semble toutefois possible d'affirmer que dans des proportions qui restent certes beaucoup plus faibles, que chez le ruminant, le broyage et l'agglomération de la ration diminuent l'activité cellulolytique dans le gros intestin du cheval et en particulier le cæcum avec vraisemblablement en contrepartie une meilleure utilisation des aliments dans l'intestin grêle.

Journées Ingestion-Digestion-Absorption de I'Associotion française de Nutrition, Paris, 15-16 novembre 1979.

Remerciements. - Nous tenons à remercier M. Candau, Maître de Conférences à la Chaire de Zootechnie de l'E. N.S. A. de Toulouse, pour l'aide apportée en ce qui concerne la pose des canules sur les poneys. 


\section{Références}

ALEXANDER F., 1972. Some functions of the large intestine of the horse. Quart. J. exp. Physiol., 37, 205. ARGENZIO R. A., SOUTHWORTH M., STEVENS C. E., 1974. Sites of organic acid production and absorption in the equine gastrointestinal tract. Am. J. Physiol., 226, 1043-1050.

HINTZ H. F., HOGUE D. E., WALKER E. F., LOWE J. E., SCHRYVER H. F., 1971. Apparent digestion in various segments of digestion tract of ponies fed diets with varying roughage grain ratios. J. anim. Sci., 32, 235-248.

KERN D. L., SLYTER L. L., WEAVER J. M., LeFFEL E. C., SAMUELSON G., 1973. Pony cecum v. s. steer rumen, the effect of oats and hay on the microbial ecosystems. J. anim. Sci., 37, 463-469.

KOLB E., GURTLER H., 1971. Ehrnährungphysiologie der landwirtschaftlichen Nutztiere. 1 vol. 957 pp. VEB Gustav Fischer Verlag, lena.

ROBINSON D. W., SLADE L. M., 1974. The current statuts of knowledge on the nutrition of equine. J. anim. Sci., 39, 1045-1066.

TISSERAND J. L., MASSON C., 1976. Effet de différents régimes sur l'activité biochimique dans le gros intestin du poney. 27e Réun. annu. Féd. eur. Zootech., Zürich, 23-26 août.

TISSERAND J. L., MASSON C., OTTIN PECCHIO, M. CREUSOT, 1977a. Mesure du pH et de la concentration en AGV dans le cæcum et le côlon du poney. Ann. Biol. anim. Bioch. Biophys., 17, 533-537.

TISSERAND J. L., CANDAU M., HOUISTE H., MASSON C., 1977b. Evolution de quelques paramètres physico-chimiques du contenu cæcal d'un poney au cours du nycthémère. Ann. Zootech., 26, 429-434.

TISSERAND J. L., 1979. L'alimentation pratique du cheval. 1 vol. 87 pP., Ed. A. Lesson. Diff. Maloine S. A. Paris.

WOLTER R., 1975. L'alimentation du cheval. $2^{\mathrm{e}}$ éd., 1 vol., 180 pP. Vigot Frères, Paris. 